\title{
Evaluation of Measles Surveillance Systems in Ginnir District, Bale Zone, Southeast Ethiopia: A Concurrent Embedded Mixed Quantitative/Qualitative Study
}

Falaho Sani ( $\sim$ falahsani@gmail.com )

Negelle Borena Health Science College

Mohammed Hasen

East Bale Zonal Health Office

Mohammed Seid

West Harerghe Zonal Health Office

Nuriya Umer

Oromia Regional Health Bureau

\section{Research Article}

Keywords: Surveillance system, Evaluation, Measles, Bale zone, Ethiopia

Posted Date: December 8th, 2020

DOl: https://doi.org/10.21203/rs.3.rs-115297/v1

License: (c) (1) This work is licensed under a Creative Commons Attribution 4.0 International License.

Read Full License 


\section{Abstract}

Background: Public health surveillance systems should be evaluated periodically to ensure that the problems of public health importance are being monitored efficiently and effectively. Despite the widespread measles outbreak in Ginnir district of Bale zone in 2019, evaluation of measles surveillance system has not been conducted. Therefore, we evaluated the performance of measles surveillance system and its key attributes in Ginnir district, Southeast Ethiopia.

Methods: We conducted a concurrent embedded mixed quantitative/qualitative study in August 2019 among 15 health facilities/study units in Ginnir district. Health facilities are selected using lottery method. The qualitative study involved purposively selected 15 key informants. Data were collected using semi-structured questionnaire adapted from Centers for Disease Control and Prevention guidelines for evaluating public health surveillance systems through face-to-face interview and record review. The quantitative findings were analyzed using Microsoft Excel 2016 and summarized by frequency and proportion. The qualitative findings were narrated and summarized based on thematic areas to supplement the quantitative findings.

Results: The structure of surveillance data flow was from the community to the respective upper level. Emergency preparedness and response plan was available only at the district level. Completeness of weekly report was $95 \%$, while timeliness was $87 \%$. No regular analysis and interpretations of surveillance data, and the supportive supervision and feedback system was weak. The participation and willingness of surveillance stakeholders in implementation of the system was good. The surveillance system was found to be useful, easy to implement, representative and can accommodate and adapt to changing conditions. Report documentation and quality of data was poor at lower level health facilities. Stability of the system has been challenged by shortage of budget and logistics, staff turnover and lack of update trainings.

Conclusions: The surveillance system was acceptable, useful, simple, flexible and representative. Data quality, timeliness and stability of the system were attributes that require improvement. The overall performance of measles surveillance system in the district was poor. Hence, regular analysis of data, preparation and dissemination of epidemiological bulletin, capacity building and regular supervision and feedback are recommended to enhance performance of the system.

\section{Background}

Public health surveillance is the continuous and systematic collection, analysis, interpretation, and dissemination of data regarding to the diseases or health-related events that have a significant public health importance, and use in public health action [1]. The purpose of evaluating public health surveillance systems to monitor efficiency and effectiveness of the system and recommendations for improving quality [1-3]. Public health surveillance system has been developed to address a range of public health needs and include a variety of data sources essential to public health action $[1,4]$. The data 
disseminated by a public health surveillance system are useful for immediate public health action, planning, to measure the burden of diseases, monitor trends, detection of outbreaks, prioritize the allocation of resources, implementation and evaluation of control programs [2-4]. Public health surveillance is carried out through a system in the health sector extending from the central level down to the peripheral health facilities up to the community level, and upward reporting and down ward feedback mechanism through sets of communication channels, which include $[1,5]$.

To ensure rapid detection of public health threats and prompt response to and recovery from various public health emergencies, the Ethiopian Public Health Institute designed a fully integrated, all-hazards, adaptable and all health approach national preparedness and response system, called Public Health Emergency Management (PHEM) system [5]. PHEM is the process of emergency preparedness, early detection, response and recovery from the consequences of public health threats to minimize the health and economic impacts from emergencies. Building a strong PHEM process and strengthening its capacity will ensure the proper implementation of International Health Regulation (IHR 2005) [5]. Nationally, 23 priority diseases/conditions that should be reported on immediately and weekly bases are selected and included into the routine surveillance system. Among those diseases under the surveillance, measles is one of immediately reportable priority diseases which have high epidemic potential [5].

Measles is endemic in Ethiopia with outbreak reported every year [6]. A total of 7,445 confirmed measles cases were reported from Ethiopia from 2016 to 2018 [5]. In the years 2019, a total of 8,202 suspected measles cases were reported from Oromia, Amhara, Afar and Somali region of Ethiopia [8]. Additionally, there was widespread measles outbreak which affected 1,043 population with 5 deaths in Ginnir district of Bale zone in 2019 and the outbreak lasted for the five months [9].

The overall purpose of surveillance is to monitor the trends over time, detection of the occurrence, identify the deviation from the threshold at the earliest point and have prompt response. Furthermore, as an early warning system, it guides prevention of the disease before the occurrence of outbreak $[5,10]$. To ensure that the public surveillance system is meeting its objective, its evaluation should involve an assessment of system attributes, including simplicity, level of usefulness, flexibility, data quality, acceptability, predictive value positive, representativeness, timeliness, and stability [1].

Thus, the public health surveillance system should be evaluated periodically to determine how well the system is operating $[1,10]$. However, the data on the performance of surveillance system are very limited in the country, particularly in the study area. In addition, despite widespread outbreak of measles in 2019 in the district, the performance of the surveillance system was not evaluated. Therefore, we evaluated the performance of measles surveillance system and its key attributes in Ginnir district, Bale zone, Southeast Ethiopia.

\section{Methods}

\section{Study setting}


The study was conducted in Ginnir district of Bale zone, which is located at a distance of $122 \mathrm{~km}$ from Robe town, the capital of Bale zone, and $557 \mathrm{~km}$ to the Southeast of Addis Ababa, the capital city of Ethiopia. Based on the 2007 national census projection, the estimated population of the district in 2019 was 164,702 . Of these 83,998 (51\%) were females [11]. Administratively, the district has 32 rural kebeles (the lowest administrative unit within the district) and has 8 health centers and 32 health posts, that provide primary health care service to the community including Public Health Emergency Management (PHEM). No private health facility available in the district. PHEM department is coordinated by one PHEM officer at the district level and responsible for coordinating the implementation of PHEM activities in health facilities under their catchment. Each health center has one PHEM focal person and the health post which is staffed by at least two Health Extension Workers (HEWs) implement PHEM activities at the lower community level.

\section{Study design and period}

We conducted a concurrent embedded mixed study approach of both quantitative and qualitative methods in August 2019, using 2018 as the base year. A cross-sectional study was carried out to determine the performance of surveillance system in the district. The qualitative interview was carried out with PHEM focal from the district health office and health facilities.

\section{Sample size and techniques}

A total of 15 study units/sites were included in the study. We included the district health office, and out of eight health centers in the district, four (50\%) were selected using lottery method. Then, out of 32 health posts, we included 10 (31\%) health posts under the catchments of the selected health centers. There are the four satellite health posts under each health center and we included at least two from each cluster health center using lottery method (Fig. 1). For the qualitative interview, a purposive sampling technique was employed to select the key informants. The key informants were the district PHEM officer, the four health centers PHEM focal person and ten HEWs from the health posts. As a result, the qualitative part involved 15 key informants into the study. At the health post level, where PHEM activities implemented by at least two health extension workers, we interviewed the most senior HEWs considering as a rich source of information.

\section{Data collection}

Data were collected using semi-structured questionnaire adapted from the Centers for Disease Control and Prevention (CDC) updated guidelines for evaluating public health surveillance systems through faceto-face interview of key stakeholders and record reviews (Additional file 1) [1]. For the quantitative survey, source of data were surveillance reporting formats, records and documents in the district health office and health facilities. For the qualitative study, the district PHEM officer, health centers PHEM focal personnel and health extension workers from health posts were interviewed using a semi-structured interview guide with flexible probing technique. Data were collected by two field epidemiologists under the supervision of principal investigator. Interviews of the participants were continued until saturation was 
reached, meaning the investigator agreed that there was redundancy in the responses and there was no new idea emerging. The questionnaire included information regarding to the operation of surveillance, communication and reporting system, case detection, availability of surveillance guideline, reporting format and documentations, case detection and registration, data analysis and interpretation, epidemic preparedness, outbreak investigation, supervision and feedback system, and questions on each surveillance attributes such as level of usefulness, completeness, timeliness, simplicity, flexibility, data quality, acceptability, predictive value positive, representativeness, and stability.

\section{Data quality assurance}

A two days training was provided to the data collectors on the overall data collection procedure. The reports and records obtained from health facilities were cross-cheeked with the documented data at the district health office to check the consistency and accuracy of the data. During the session of each visit, we briefed the stakeholders about the purpose of the assessment, which was to evaluate the performance of the system rather than individual performances. Prior to data collection, discussion was made with stakeholders from Bale zonal health department, Ginnir district health office and the health facilities, to ensure that the evaluation of the system addresses appropriate questions and attributes, to produce useful and acceptable findings.

\section{Data analysis}

The quantitative data were entered and analyzed using Microsoft Excel 2016. All questionnaire responses were dichotomized except for the open-ended questions. Quantitative findings were summarized by their frequency and proportion. Qualitative data were analyzed manually using thematic analysis. Data were cleaned before analysis and the qualitative findings were narrated and summarized based on thematic areas to supplement the quantitative results.

\section{Operational definitions}

\section{Usefulness}

A public health surveillance system is useful if it contributes to the prevention and control of adverse health-related events, including an improved understanding of the public health implications of such events. It is measured by the actions taken as a result of analysis and interpretation of the data from the public health surveillance system [1].

\section{Completeness}

The proportion of health facilities that submitted a report to the higher level, out of expected facilities irrespective of the time of submission [1].

\section{Timeliness}

The timeliness of the report was assessed at two level. First, calculated by assessing how many of its expected reports have submitted to the next level within the prescribed time. Second, the time interval 
between the onset of health-related event and the reporting of the event the, and the time required for the identification outbreaks or the effect of control and prevention measures [1].

\section{Simplicity}

Refers to the structure of the system and the ease of implementation while still meeting its objectives [1].

\section{Flexibility}

The ability of the system to adapt to changing information needs or operating conditions with little additional time, personnel, or allocated funds [1].

\section{Data quality}

Data quality assessed based on content completeness of the reporting formats and validity of the data recorded [1].

\section{Acceptability}

Willingness of surveillance stakeholders to implement the system as expressed by their active participation in case detection and reporting [1].

\section{Predictive value positive}

The proportion of cases detected by the surveillance case definition that actually have the disease [1].

\section{Representativeness}

The ability of the system to describe the occurrence and distribution of a health-related event accurately in terms of time, place and person, and also related to the health service coverage and the reporting rate of the health facilities [1].

\section{Stability}

Refers to the reliability (i.e., the ability to collect, manage, and provide data properly without failure) and availability of the public health surveillance system which is the ability to be operational when it is needed [1].

\section{Results}

\section{Operation of surveillance, communication and reporting system}

The routine flow of surveillance data is usually from reporting sites to the next level up to the central level. The health posts prepare and send their weekly surveillance report to the cluster health centers through a 
phone call every Monday in the morning. On the same days at afternoon, the health centers in turn aggregate the surveillance report received from the health posts under their catchment send to Ginnir district health office. Similarly, the district health office receives reports from health centers using phone call and sends to Bale Zonal Health Department on Tuesday afternoon (Fig. 2). The surveillance system is fully integrated into the routine health care delivery system and implemented throughout the year without interruption. The community and health facilities especially health posts are the main source of information. The report submission to the higher level was through a phone call, followed by the paperbased report submission. All the four health centers and ten health posts has no wired phone and they communicate with the next level with their mobile phone. The district health office has a wired office phone and Wi-Fi network. As represented in Fig. 2, the flow of surveillance data and information is from the community to a higher level, whereas supervision and feedback follow the reverse direction.

\section{Availability of surveillance guidelines, reporting formats and documentation}

The national PHEM guideline was available at the district level and in all visited health centers. However, out of ten health posts, only three (30\%) of the visited health posts had national PHEM guideline manual. There was shortage of standard weekly reporting format in the visited health centers and health posts. Out of four health centers and ten health posts, the weekly reporting format was available in $2(50 \%)$ of health centers and $8(80 \%)$ of the health posts. In addition, the case-based reporting format was also not available in the visited health facilities. However, weekly surveillance reporting format and measles casebased reporting format is available at Ginnir district health office. Regarding the documentation of weekly surveillance report, all health centers and the district health office had a copy of each week report in the file cabinet. However, copy of weekly surveillance report is not available at the visited health posts.

\section{Case detection and registration}

All health facilities and the district health office had the case definition for measles diseases.

Understanding of the measles case definition at the visited health centers and district health office was good as explained by PHEM focal persons. But, at the health post level only 4 health extension workers (40\%) well understood the measles case definition. Among the visited health facilities, all health centers and $3(30 \%)$ of the health posts were posted the measles cases definition on a wall or notice board. Health care workers has been detecting suspected cases of measles using standard case definitions. All visited health centers and only three (30\%) of health posts were using clinical register and correctly recording the reportable diseases including measles. In addition, no rumor registration logbook available at the visited health facilities.

\section{Data analysis and interpretation}

All health centers had at least one computer in addition to the computer for the health management information system. However, none of the health centers had using for the analysis of surveillance data. The health centers send their surveillance weekly report which includes only the total number of cases 
and performed activities to the district health office, without analysis and interpretation of surveillance data. The district health office and all health facilities had denominators which helps to describe the surveillance data epidemiologically in terms of person, place and time such as total number of populations disaggregated by age group, sex and kebeles, etc. However, the district health office simply aggregates the reports received from the health centers and sends zonal health department without performing analysis of surveillance data. In addition, there is no monitoring of accumulation of susceptible individual for measles over time using measles risk assessment tool by the district health office even if data on vaccination coverage and annual birth cohort available.

\section{Epidemic Preparedness and Management}

Ginnir district health office has Emergency Preparedness and Response Plan (EPRP), but there is no allocated budget to respond in case an emergency occurred. The health facilities didn't have a written EPRP and outbreak investigation checklist. Stocks of emergency drug and supplies for epidemic response were not available both at the district health office and health facilities, however whenever an outbreak exists, the health centers mobilize drugs and other medical supplies from their store which was reserved from the routine services. In addition, the district health office mobilizes emergency drug and supplies from zonal health department every time outbreak occurred. The district was established a Rapid Response Team (RRT) and Multisectoral PHEM task force (Epidemic Management Committee) which is coordinated by woreda chief administrator; however, they are activated only when the outbreak exists. There are no documented meeting minutes by the committee. There was no vehicle assigned specifically to the public health emergency management department at the district level. The PHEM focal person from district health office and health centers had received training on basic PHEM in the year 2018. However, out of 10 health extension workers interviewed, only $2(20 \%)$ of them received basic PHEM training.

\section{Outbreak investigation}

In the year 2019, there was a widespread measles outbreak in Ginnir district and affected 1043 population with 5 deaths. According to the district PHEM focal, the outbreak was lasted for five months and affected all the 32 kebeles in the district. The sample of five suspected cases were collected and confirmed by laboratory investigation at the national public health institute. The district was encountered shortage of emergency drugs and supplies during the outbreak. The cases were recorded on the line list and analyzed by time, place and person according to the district PHEM focal. However, there was no documented report or standard procedures available for investigation of the measles outbreak. In addition, there was no assessment of the lesson learnt from the outbreak and post epidemic evaluation conducted by epidemic management committee to prevent the occurrences of outbreak in the future.

\section{Supportive supervision and feedback}

Both the district health office and health centers had a supervision plan, but as stated by surveillance focal it was not conducted regularly. All study sites didn't have a supervision checklist to assess PHEM activities including measles surveillance in detail. However, there was an integrated checklist which 
includes only few data elements regarding to PHEM at the district health office and health center. The district PHEM department has been supervised by zonal once in the year, but no written feedback of supervision was provided to the district. The Ginnir districts PHEM department had conducted two supportive supervision to health centers on integrated activities including surveillance. Out of ten health posts, only $6(60 \%)$ were supervised by the health centers regarding to surveillance activities in 2018. Despite this, there was no written letter of feedback provided to the district PHEM department and health facilities from the respective higher level. The common reason for poor supervision activities were, shortage of manpower, lack of resource, poor coordination and work overload according to the PHEM personnel. District PHEM department and health centers provides only verbal feedback during quarterly and monthly meeting. In general, no regular supervision and feedback system, weekly bulletin preparation and dissemination regarding to public health surveillance in the district and health facilities.

\section{Attributes of surveillance system \\ Level of usefulness}

According to the respondents, the surveillance system is very helpful to determine the magnitude of morbidity and mortality of diseases under surveillance as well as to assess the effectiveness of prevention and control measures for the priority diseases including measles. For the surveillance officers, the system helped to detect outbreaks early and take action to prevent epidemics. The system also helps to identify the priority areas for each disease under surveillance and for resource allocation. However, there is no actions taken as a result of analysis and interpretation of the data from the public health surveillance system.

\section{Completeness}

The completeness of report differs from one health facility to another health facility. The report completeness by the health centers in Ginnir district was below expected level of $80 \%$ in the WHO week 4, $9,25,28,34,35,46$ and 49 in 2018 as shown in Fig. 3. However, the average annual report completeness by health centers was $93 \%$ in 2018 . The report completeness by the health posts were below expected level of $80 \%$ in the WHO week 4, 25, 35 and 39, however, the average annual report completeness was $95 \%$ (Fig. 4). Furthermore, the average completeness of surveillance report by the district was $95 \%$ in 2018 as presented on Fig. 5.

\section{Timeliness}

In the visited health facilities and at the district level, the date report received was not recorded on the report, as a result it was difficult to calculate the report timeliness. However, the average surveillance report timeliness calculated by the Ginnir district health office in the year 2018 was $87 \%$. Another aspect of timeliness is the time required for the identification of outbreaks or the effect of control and prevention measures, and the time interval between the onset of health-related event and the reporting of the event. According to the district PHEM officer, the recent measles outbreak occurred in 2019 was reported to the 
zonal health department after two weeks of confirmation by the district. The intervention for control of outbreak was also initiated by the district after one month of confirmation due to shortage of resources. Additionally, the outbreak response immunization targeting children aged 6 months to 14 years was also initiated after two months.

\section{Simplicity}

All respondents agreed that measles case definitions for identification of suspected cases are clear and easy to understand. Measles standard case definition was mostly used at the health center level, while community case definition which is simplified and translated to the local language Afan Oromo is used by the health extension workers at the community level. All stakeholders stated that the route of surveillance data flow is clear and they are familiar with when and to whom the report sent. The surveillance reporting formats are also easy to complete the data and also well understood by all respondents including health extension workers. The time to fill the weekly surveillance report is 20-25 minutes by district PHEM officer, while it takes only 15-20 minute by health centers PHEM focal and health extension workers.

\section{Flexibility}

The district PHEM officer and health facilities respondents agreed that the current weekly surveillance reporting format can accommodate new health events. They also explained that implementation of national PHEM guideline will not be difficult with changes in existing procedure, case detection and report forms.

\section{Data quality}

Even though the majority of the fields in the reporting format were correctly filled, we have observed the following gaps related to the data quality; blank spaces that should be filled with zero (0) number were not recorded not recorded, the starting and ending dates of the week were not also completely filled. In addition, the date report received and sent is important variables to determine timeliness of the reporting, but commonly missed at the district and health facility level.

\section{Acceptability}

The surveillance officials have been actively participated in reporting as indicated by $95 \%, 93 \%$ and $95 \%$ achievement in the average report completeness by the district health office, health centers and health posts respectively. All health centers and health posts have been using the standard measles case definition for the detection of suspected cases, and appropriately using the reporting formats. Hence, the surveillance system was acceptable by district PHEM officer, health centers PHEM focal persons and health extension workers.

\section{Predictive value positive}

Calculating the predictive value positive (PVP) of measles require the records of information obtained from the public health surveillance system. A record of the number of suspected measles cases detected 
by surveillance system and those confirmed to have measles by the laboratory investigation of Immunoglobulin M (IgM) is required to calculate the PVP for measles surveillance. As a result of incomplete documentation of this data at district and health facility level, it was difficult to calculate PPV. However, during the measles outbreak in 2019 , out of collected five samples from the suspected measles cases and tested at the national laboratory, all were found to be positive for IgM. In thisregards, the predictive value positive for measles surveillance was $100 \%$.

\section{Representativeness}

The measles surveillance report has been reported from the lowest community level to the health posts, and also from the disease registries at health posts and health centers. However, the weekly surveillance reporting format lacks some important epidemiological variables like sex and age, which is vital to describe the distribution of diseases by personal characteristics and help to generate information for appropriate actions. Regarding to the health service coverage, one health center and health post is expected to serve 25,000 and 5,000 population respectively. A total of eight health centers and 32 health posts available in the Ginnir district for the total population of 164,702 . Therefore, the health service coverage in the district were above $100 \%$ by the health center and $97 \%$ by the health posts. Furthermore, the surveillance report was reported from all health facilities in the district.

\section{Stability}

Availability of PHEM focal person both at the district and health facility level was enabled the surveillance system to be operational even with a limited resource. Of the 15 respondents interviewed, majority 12 (80\%) explained that, the shortage of budget and logistics specific to the surveillance system and staff turnover were the common factors affecting the stability and well-functioning of the system to achieve its intended objectives and purpose. Furthermore, the surveillance system was integrated to the routine health care delivery and operational throughout the year.

\section{Discussion}

Public health surveillance system need to be evaluated periodically to determine how well the system is operate and the evaluation should emphasize attributes that are most important for the objectives of the system [1]. We evaluated the performance of measles surveillance system in Ginnir district, Southeast Ethiopia.

The surveillance system is fully integrated into the routine health care delivery system and have been implemented throughout the year. The community and health facilities in the districts the main sources of information. The structure of data flow was from the community at the bottom to the respective upper level. The flow of data was well organized and the reporting facilities are well familiar with the line of communication. Nevertheless, the flow of surveillance reporting and communication has several challenges such as lack of wired telephone and internet access for regular communication. 
Effective public health responses depend on the ability of the surveillance system to provide reliable, timely and complete information to support action $[10,12]$. According to the findings of our study, the weekly surveillance report completeness of health centers and health posts were $93 \%$ and $95 \%$ respectively in 2018. Similarly, the overall report completeness of the district was $95 \%$. The report completeness in our finding is higher than the $90 \%$ national target set by the country by the year $2019 / 20$ [13], and the target of $80 \%$ set by the world health organization [10]. Our finding is also consistent with evaluation of public health surveillance system in Dangila district, Northwest Ethiopia which reported the surveillance report completeness higher than the national target [14]. This indicates acceptance of the public health surveillance system by all stakeholders engaged in the system.

The average surveillance report timeliness by the district was $87 \%$, which is higher than the target of $80 \%$ set by the world health organization [10], and the $61 \%$ timeliness report from study in Northern Ghana [15]. However, the report timeliness in our study is lower than the $95 \%$ national target set nationally by the health sector transformation plan in 2019/20 [13], and the $94.6 \%$ report timeliness reported from the of study conducted in Dangila district, Northwest Ethiopia [14]. In addition, the district health office was not timely notified the measles outbreak occurred in 2019 to zonal health department, and the intervention activities to control the outbreak was also initiated late. Effective communication channel and timely reporting of surveillance data is required to provide timely information, which will help for early detection of health-related events and immediate action $[5,7,10]$. The lower report timeliness in the study area might be due to lack of regular communication line between the reporting site and higher level as indicated by absence of wired office telephone service and internet access.

According to the national guideline, surveillance data analysis and interpretation should be done weekly at each level where the data are collected, starting from health facility to national level [5]. The health centers in Ginnir district aggregate the data they received from health posts and send it to the district health office every Monday in the afternoon. However, the data are analyzed on weekly, monthly and quarterly bases, and described by time, place and person having the denominators like total population disaggregated by sex, age and kebeles, etc. Similarly, the district health office simply merges the aggregate reports received from the health centers and sends to the zonal health department without analysis. Furthermore, monitoring of accumulation of susceptible individual for measles over time period is crucial in prediction and prevention of measles outbreak [12], but not being monitored and analyzed by the district health office. Similar finding was reported from the study in Dangila district, Northwest Ethiopia [14]. The surveillance data must be analyzed and provide key information for decision making and public health action. The possible reasons for this area skill gap in data analysis and management, weak supervision and feedback system, poor commitment and lack of continuous capacity building through update trainings.

The district health office has Emergency Preparedness and Response Plan (EPRP) which was not supported by the budget and supplies to respond to the emergencies when occurred. The health facilities didn't have written EPRP and outbreak investigation checklist. The rapid response team and multisectoral epidemic management committee established in the district were not fully functional. Additionally, the

Page 12/23 
epidemic management committee has no documented meeting minutes, review of plans and lesson learnt by conducting post epidemic evaluation. Our finding is supported by the report of study in Dangila district, Northwest Ethiopia [14]. According to the study of measles outbreak investigation in Ginnir district, the intervention activities to control the outbreak was initiated late by the district, after one month of the outbreak and the outbreak response immunization targeting children aged 6 months to 14 years was also initiated too late after two months [9]. Poor preparedness and response to the public health emergencies have an adverse consequence for the community. The possible reason for poor functionality of rapid response team and epidemic management committee might be absence of capacity building, absence of ownership sense and poor supervision from the officials at higher level.

When outbreak is detected, it should be investigation in order to identify the source or the cause, to manage the cases, and to control and prevent further spread of the outbreak [12]. Outbreaks occur when the accumulated number of susceptible individuals is greater than the critical number of susceptible individuals for a given population to sustain transmission [12]. However, the district health office was not investigated the measles outbreak which affected 1043 population with 5 deaths in 2019 [9], using epidemiological design. Study conducted in Dangila district, Northwest Ethiopia reported similar finding [14]. According to the district PHEM department, the main reason for not investigating the outbreak was lack of capacity and skill to conduct investigation according to its procedure.

Regular supportive supervision and feedback is crucial to improve the performance of surveillance system. The Ginnir district health office and health centers were conducting a supportive supervision activity in an irregular and integrated ways, and not using the supervision checklist specific to assess the surveillance activities. Developing regular epidemiological bulletin which shows the trends of diseases over time and disseminating the bulletin for the surveillance officials is essential to build strong feedback mechanism. However, written feedback system for the performed activities was not practiced in the district. The main reason for the week feedback system in the district is due to the absence of analysis and interpretation of surveillance data, and dissemination for the health facilities. Similar finding was reported from the study conducted in Dangila district, Northwest Ethiopia [14] and study in Akwa-lbom state of Nigeria [16].

Regarding to the attributes, the surveillance system of the district was found to be useful, simple, acceptable, flexible, and representative. The quality of surveillance data was poor in recording the time period of the report and the date of report received and sent. The average surveillance report timeliness was also lower (87\%), no timely notification of outbreak and initiation of intervention by the district. The stability and functioning of the system to achieve its intended purpose have been challenged by the shortage of budget and logistics, staff turnover, absence of update training for health workers, and poor supportive supervision and feedback mechanism. Our finding is in line with the reports of study conducted in Dangila district, Northwest Ethiopia [14].

This study has clearly demonstrated the performance of measles surveillance system in Ginnir district; however, our study has some constraints. There might be socially desirable responses by the surveillance 
personnel's working within the public health surveillance due to fear of measures by the officials as a result of disclosing the actual situations. But, prior to the data collection we held discussion with the stakeholders and briefed in detail the objective of the assessment which was to evaluate the performance of the surveillance system rather than the individual's performances.

\section{Conclusions}

The surveillance system of the district found to be useful, simple, acceptable, flexible, and representative. Quality of data, timeliness, and stability of surveillance system were attributes that require improvement to enhance performance of the system. Weekly report completeness was above the target, but the timeliness of the report was below the national target. The surveillance system is fully integrated into the routine health care delivery system and the structure of surveillance data flow was from the community at the bottom to the respective upper level. However, the district surveillance system has the following gaps; poor line of communication between surveillance levels, lack of regular surveillance data analysis and interpretation, absence of EPRP at health facility level, lack of budget, poorly functional rapid response team and epidemic management committee, failure to investigate the outbreak, and weak supportive supervision and feedback system. Therefore, to strengthen the performance of surveillance system building strong line of communication, regular analysis and interpretation of surveillance data, preparation of epidemiological bulletin and dissemination to the stakeholders and regular supportive supervision and feedback is recommended for the surveillance focal of the district. Furthermore, zonal health departments are required to made functional the RRT and epidemic management committee, allocate budget for surveillance activities and provide capacity building trainings for the surveillance officials at the district and health facility level.

\section{Abbreviations}

CDC: Center for Disease Control and Prevention; CFR: Case Fatality Rate; EPRP; Emergency Preparedness and Response Plan; HEWs: Health Extension Workers; IDSR: Integrated Disease Surveillance and Response; MCV: Measle Containing Vaccine; PHEM: Public Health Emergency Management; PVP: Predictive Value Positive; RRT: Rapid Response Team; WHO: World Health Organization.

\section{Declarations}

\section{Ethics approval and consent to participate}

The study was conducted in accordance with the Declaration of Helsinki. The letter of support which was obtained from Epidemiology Department of Jimma University and Bale Zonal Health Department was submitted to the Ginnir District Health Office. Permission to use the data and conduct the study was taken from Ginnir district health office and health facilities. Written informed consent was obtained from each participant after the purpose of the study was explained. They were also informed that all the data obtained from them would be kept confidential and no individual data was included in the study. 


\section{Consent for publication}

Not applicable.

\section{Availability of data and materials}

The datasets used in this study are available from the corresponding author up on reasonable request.

\section{Competing interests}

The authors declare that they have no competing interests.

\section{Funding}

Funding was not received from any organization.

\section{Authors' contributions}

FS designed the study, supervised the data collection, analyzed the data, and wrote the first draft of the manuscript. MH participated in the design, data collection, and critical review of the manuscript. MS participated in the data collection, analysis and interpretation of the data. NU participated in the design, and reviewed and modified the drafts of the manuscript. All the authors have read and approved the final version of the manuscript. We also to appreciate all PHEM personnel's willingness and cooperation in giving us the data on the surveillance system.

\section{Acknowledgments}

We are thankful to Ethiopian Field Epidemiology and Laboratory Training Program and Epidemiology Department, Jimma University for their facilitation and support. We gratefully acknowledge Bale Zonal Health Department, Ginnir District Health Office, Health facilities for their cooperation and participation to the successful completion of this study. We would like to extend our gratitude to Mr. Desta Hiko (Associate Professor of Epidemiology, Jimma University) for his encouragement and guidance. We also appreciate all PHEM personnel for their willingness and cooperation in giving us the data on the surveillance system.

\section{Authors' information}

FS, MH, MS and NU has MPH in Field Epidemiology.

\section{References}

1. Centers for Disease Control and Prevention. Updated guidelines for evaluating public health surveillance systems: recommendations from the guidelines working group. MMWR. 2001. p. 1-35. 
2. Buehler JW, Rothman KJ, Greenland S. Modern epidemiology, 2nd edition. Philadelphia, PA: Lippencott-Raven; 1998.

3. Teutsch S, Thacker S. Planning a public health surveillance system. Epidemiol Bull Pan Am Heal Organ. 1995;16:1-6.

4. Thacker SB, Stroup DF. Future directions for comprehensive public health surveillance and health information systems in the United States. Am J Epidemiol. 1994;140(383-97).

5. Ethiopian Public Health Institute. Public Health Emergency Management Guidelines for Ethiopia. 2012.

6. World health Organization. WHO-GAVI Joint Statement: Ethiopia Launches Measles Vaccine Second Dose on 11th February 2019. Addis Ababa, Ethiopia. 2019.

7. World Health Organization. Global Measles and Rubella Update November 2018. Geneva, Switzerland; 2018.

8. Ethiopia World Health Organization. Health Cluster Bulletin \#7. August 2019.

9. Kalil FS, Gemeda DH, Bedaso MH, et al. Measles outbreak investigation in Ginnir district of Bale zone, Oromia region, Southeast Ethiopia, May 2019. Pan Afr Med J. 2020;36:20.

doi:10.11604/pamj.2020.36.20.21169

10. World Health Organization AFR REGION. Technical Guidelines for Integrated Disease Surveillance and Response in the African Region. Atlanta, Georgea: CDC; 2010.

11. Central Statistical Agency. 2007 Population and Housing Census of Ethiopia: Administrative Report; 2012.

12. Ethiopian Health and Nutrition Research Institute. Guideline on measles surveillance and outbreak management. 3rd Editio. Addis Ababa, Ethiopia. 2012.

13. Ethiopia Ministry of Health. Health Sector Transformation Plan 2015/16 - 2019/20. Ethiopia; 2015.

14. Alemu T, Gutema H, Legesse $S$. et al. Evaluation of public health surveillance system performance in Dangila district, Northwest Ethiopia: a concurrent embedded mixed quantitative/qualitative facilitybased cross-sectional study. BMC Public Health. 2019;19:1343. https://doi.org/10.1186/s12889-0197724-y

15. Adokiya MN, Awoonor-Williams JK, Beiersmann C, et al. Evaluation of the reporting completeness and timeliness of the integrated disease surveillance and response system in northern Ghana. Ghana Med J. 2016;50(1):3-8.

16. Motilewa O, Akwaowo CD, Ekanem AM. Assessment of implementation of integrated disease surveillance and response in Akwa Ibom State Nigeria. Ibom Medical Journal. 2015;8(1):24-5.

\section{Figures}




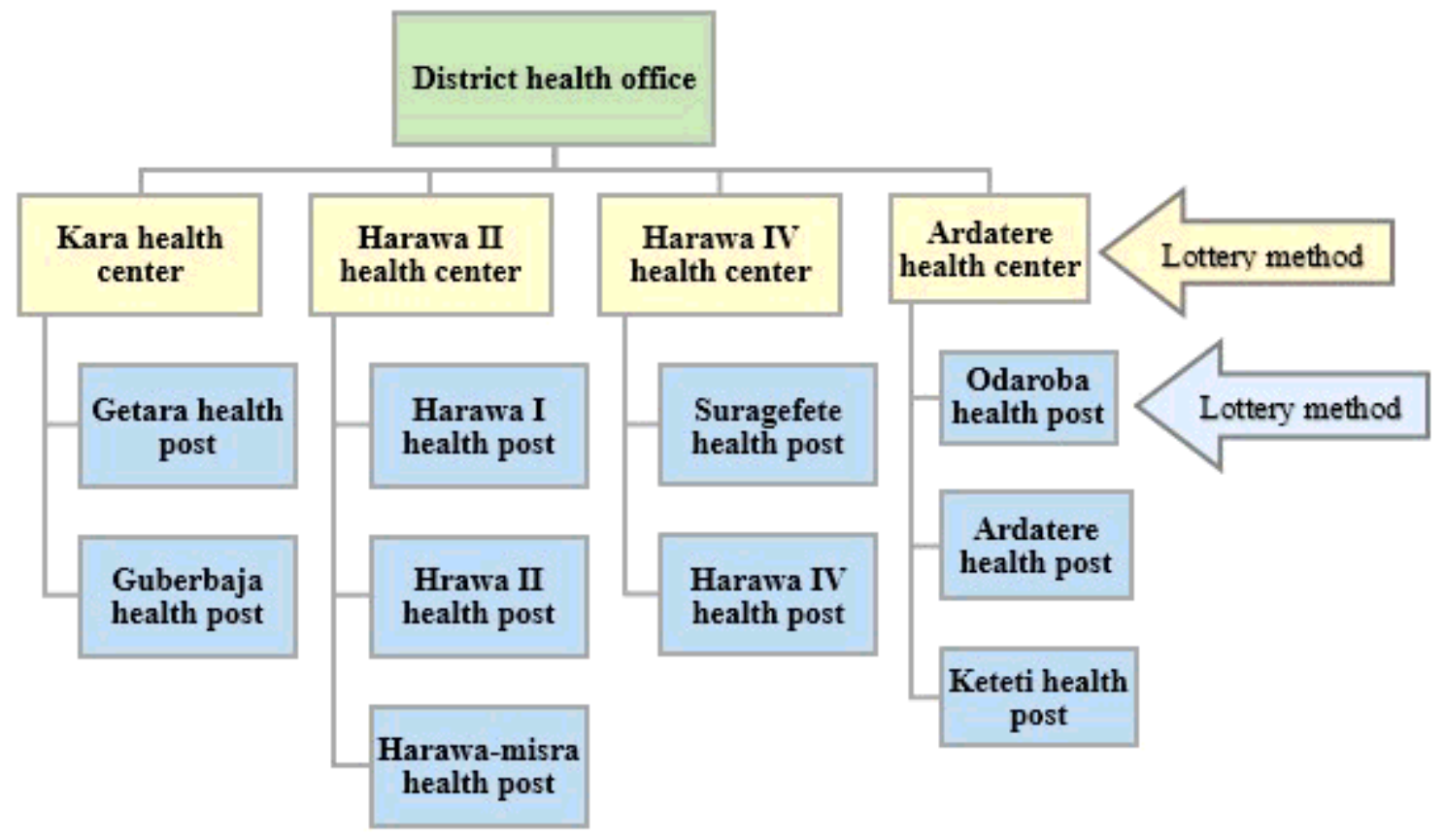

Figure 1

Schematic presentation of sampling procedure for evaluation of measles surveillance systems in Ginnir district, Southeast Ethiopia.

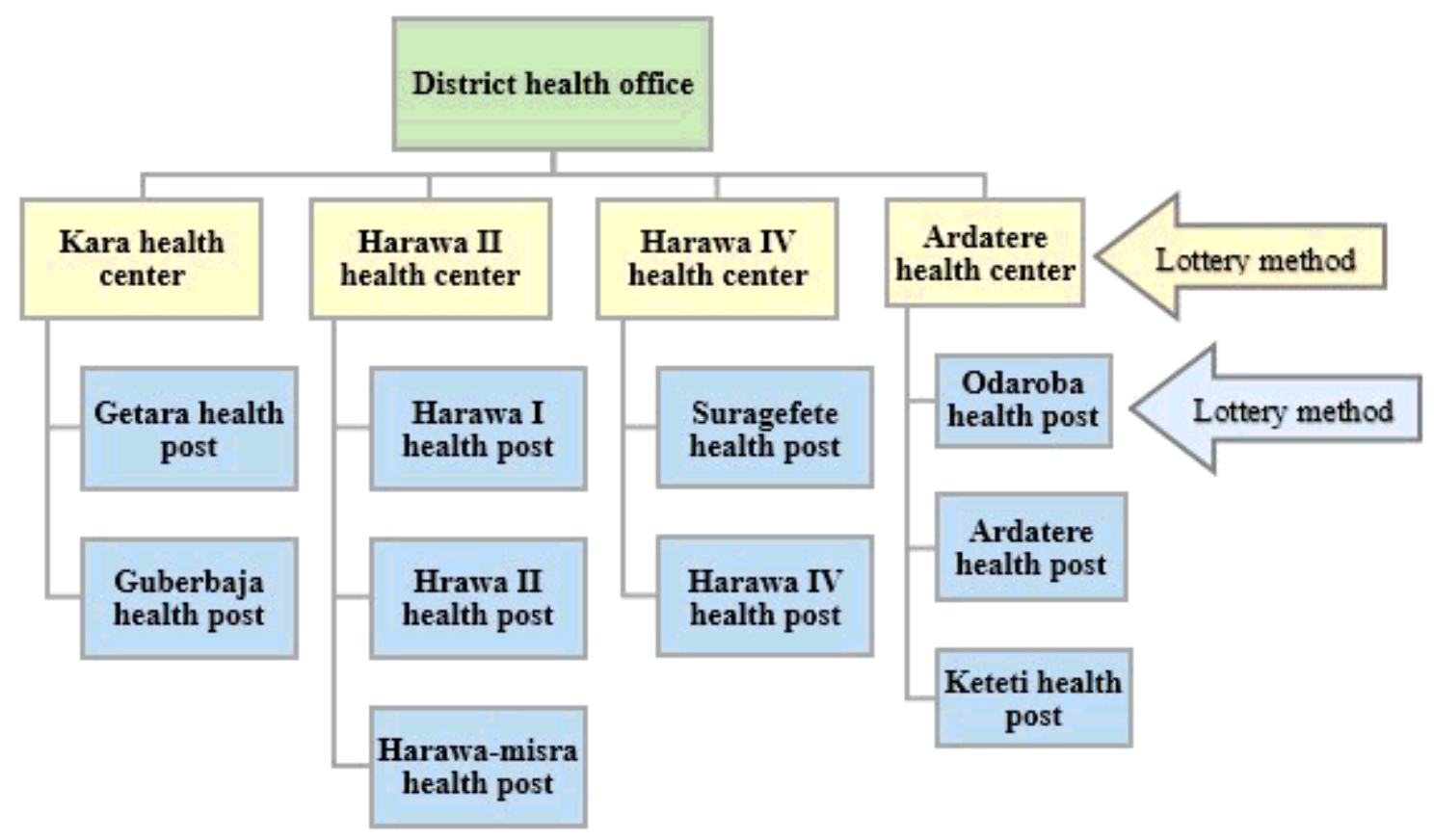

Figure 1

Schematic presentation of sampling procedure for evaluation of measles surveillance systems in Ginnir district, Southeast Ethiopia. 


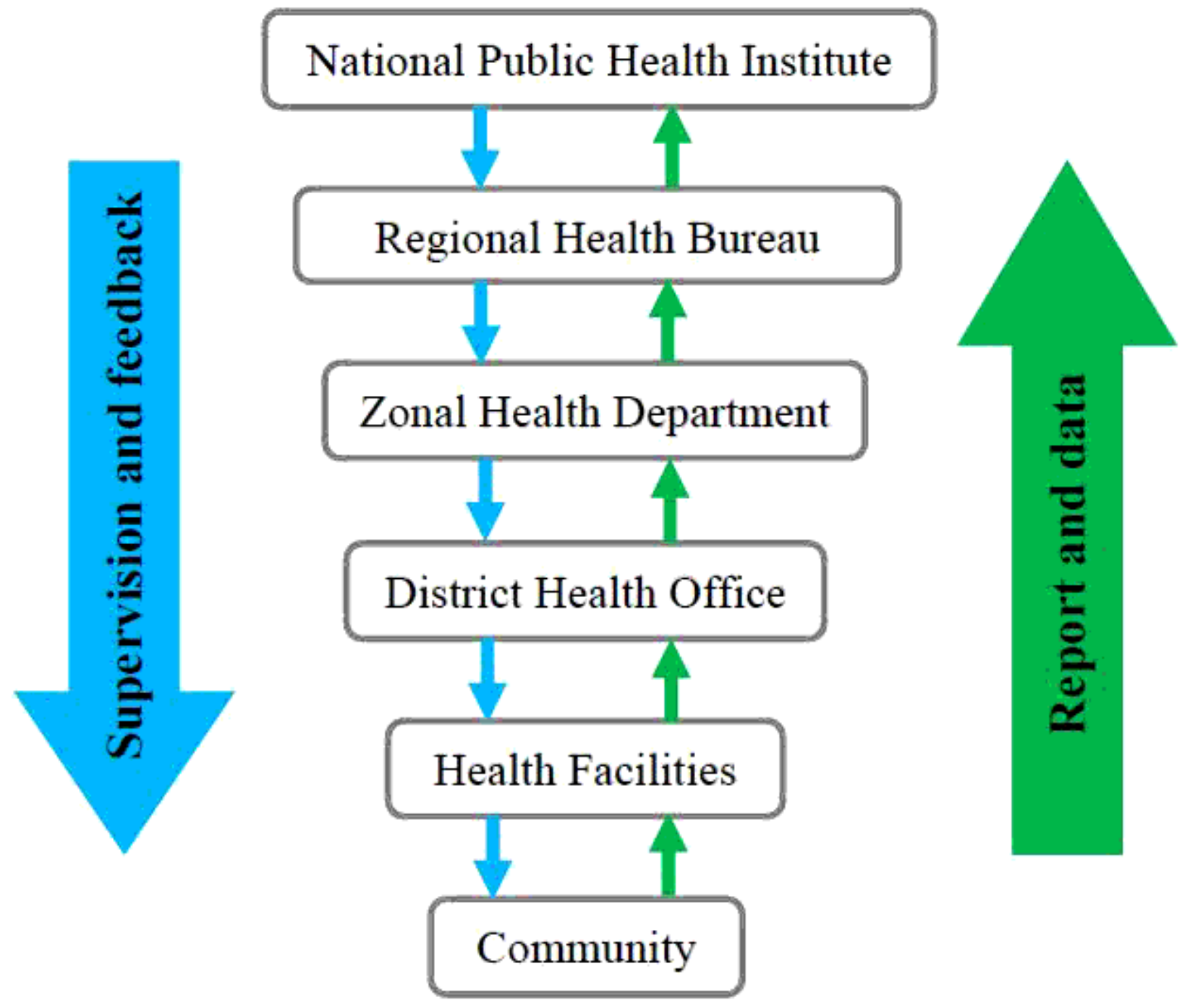

Figure 2

Diagram showing the flow of surveillance data and feedback in Ginnir District, Southeast Ethiopia. 


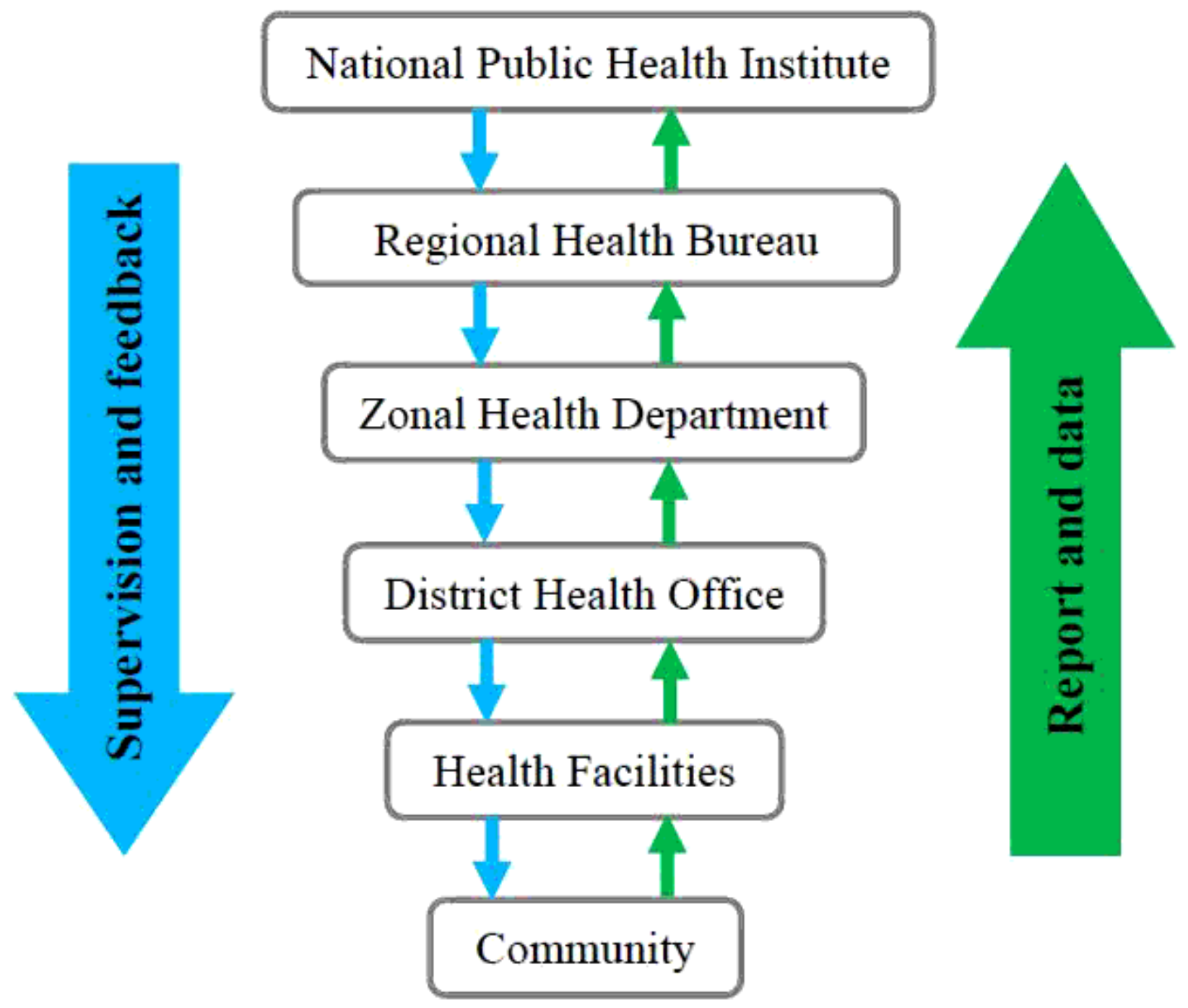

Figure 2

Diagram showing the flow of surveillance data and feedback in Ginnir District, Southeast Ethiopia. 


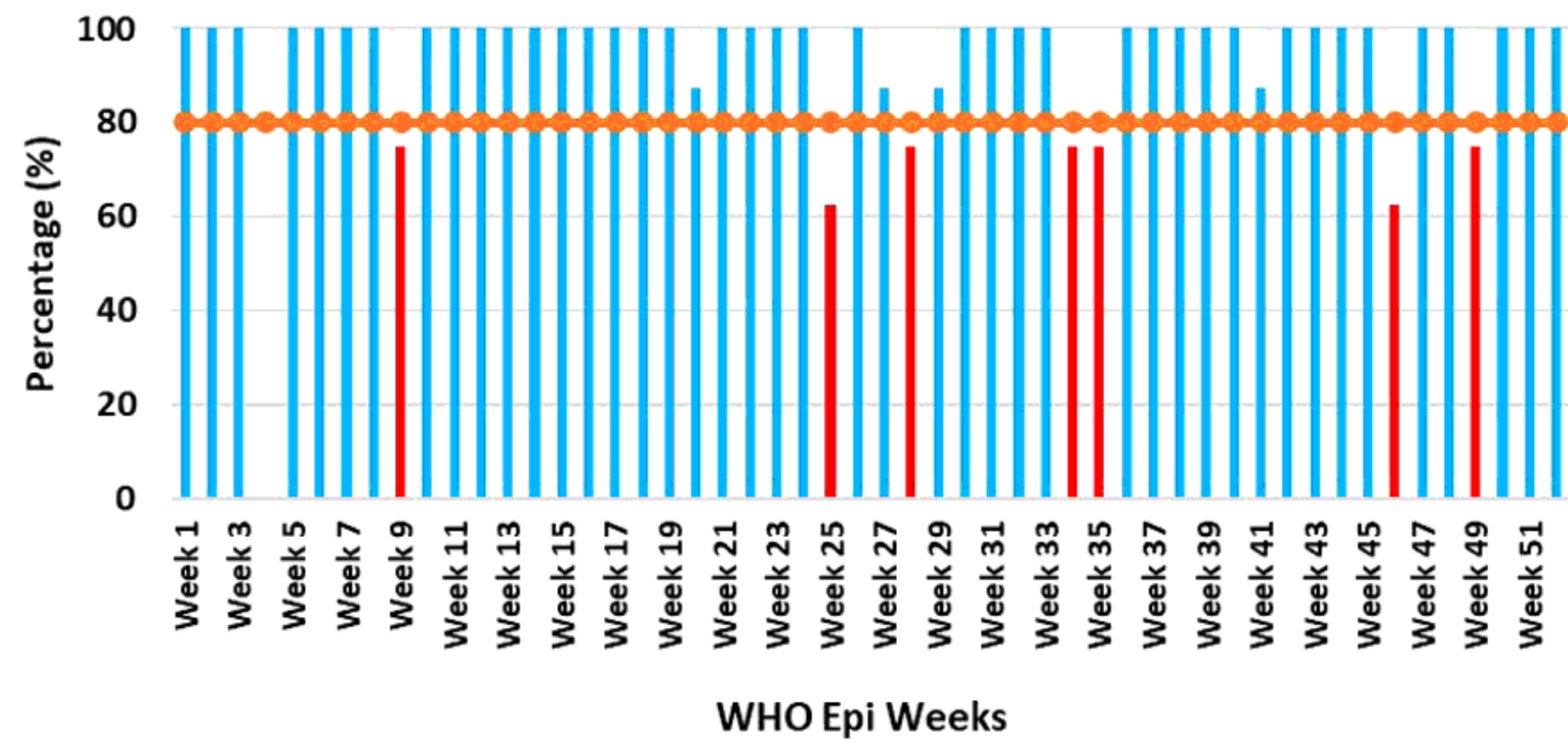

Figure 3

Completeness of health centers weekly surveillance report by WHO weeks, Ginnir district, Southeast Ethiopia.

\section{Health Center Completeness $\quad \longrightarrow$ Target}

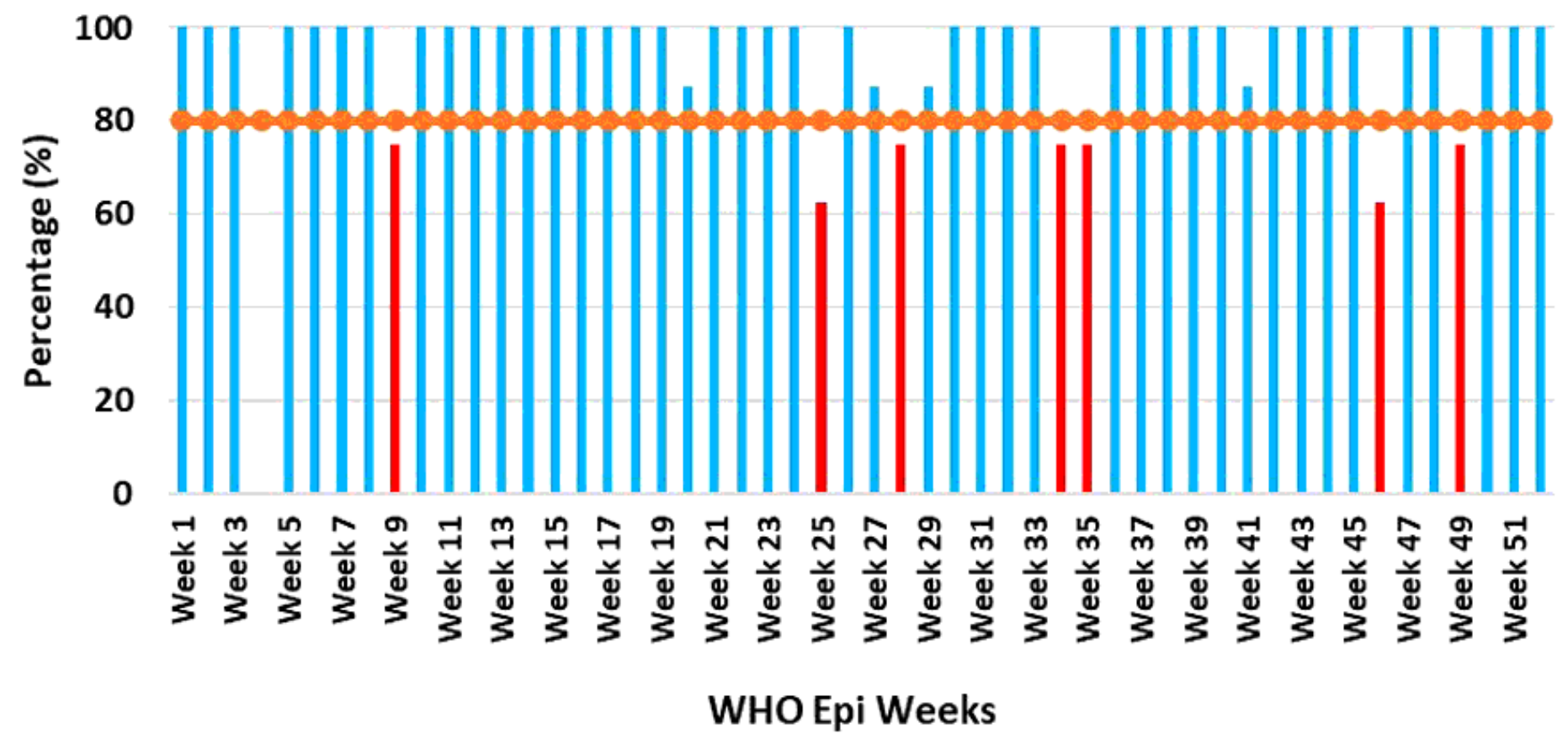


Figure 3

Completeness of health centers weekly surveillance report by WHO weeks, Ginnir district, Southeast Ethiopia.

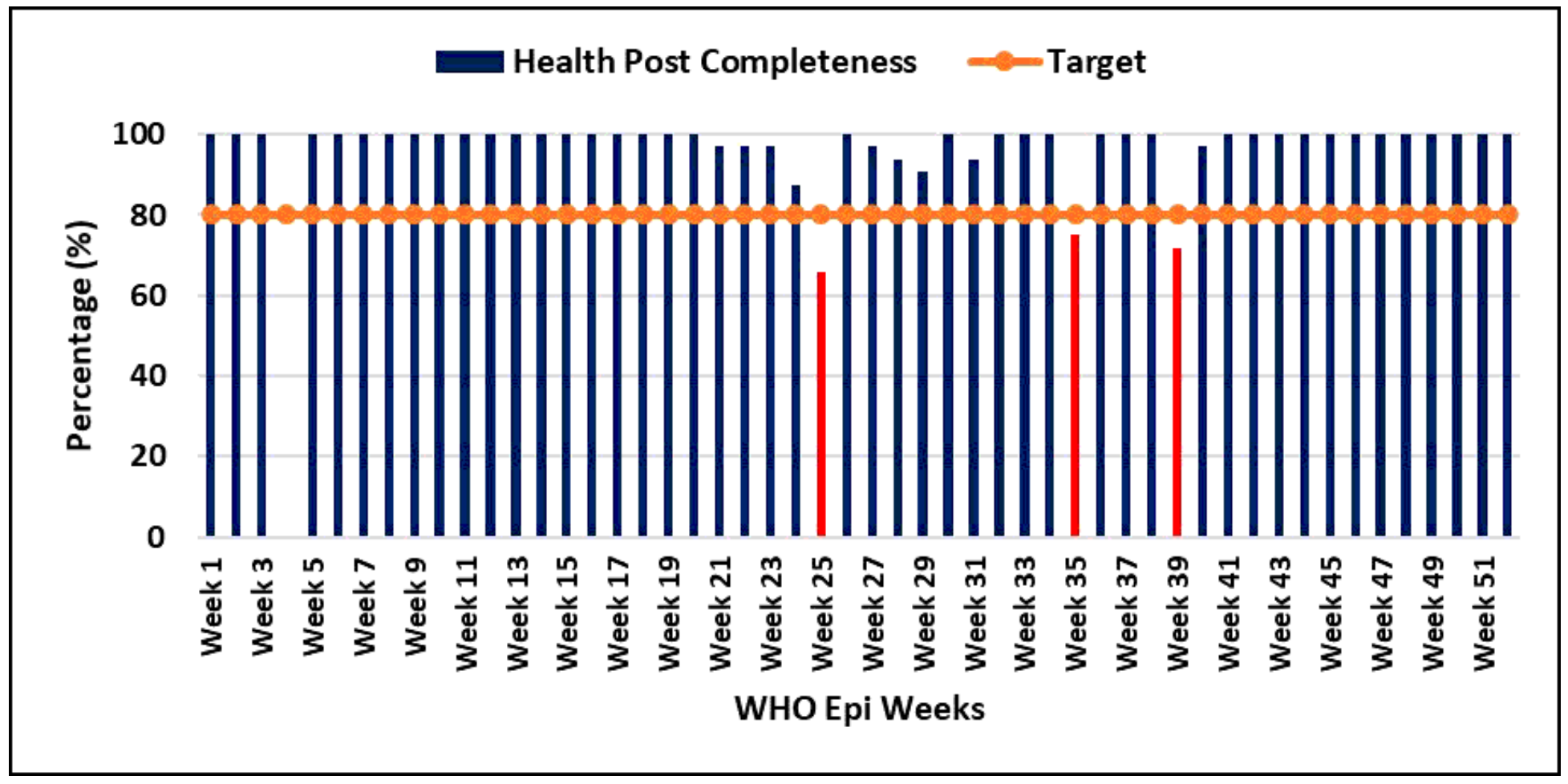

Figure 4

Completeness of health posts weekly surveillance report by WHO weeks, Ginnir district, Southeast Ethiopia.

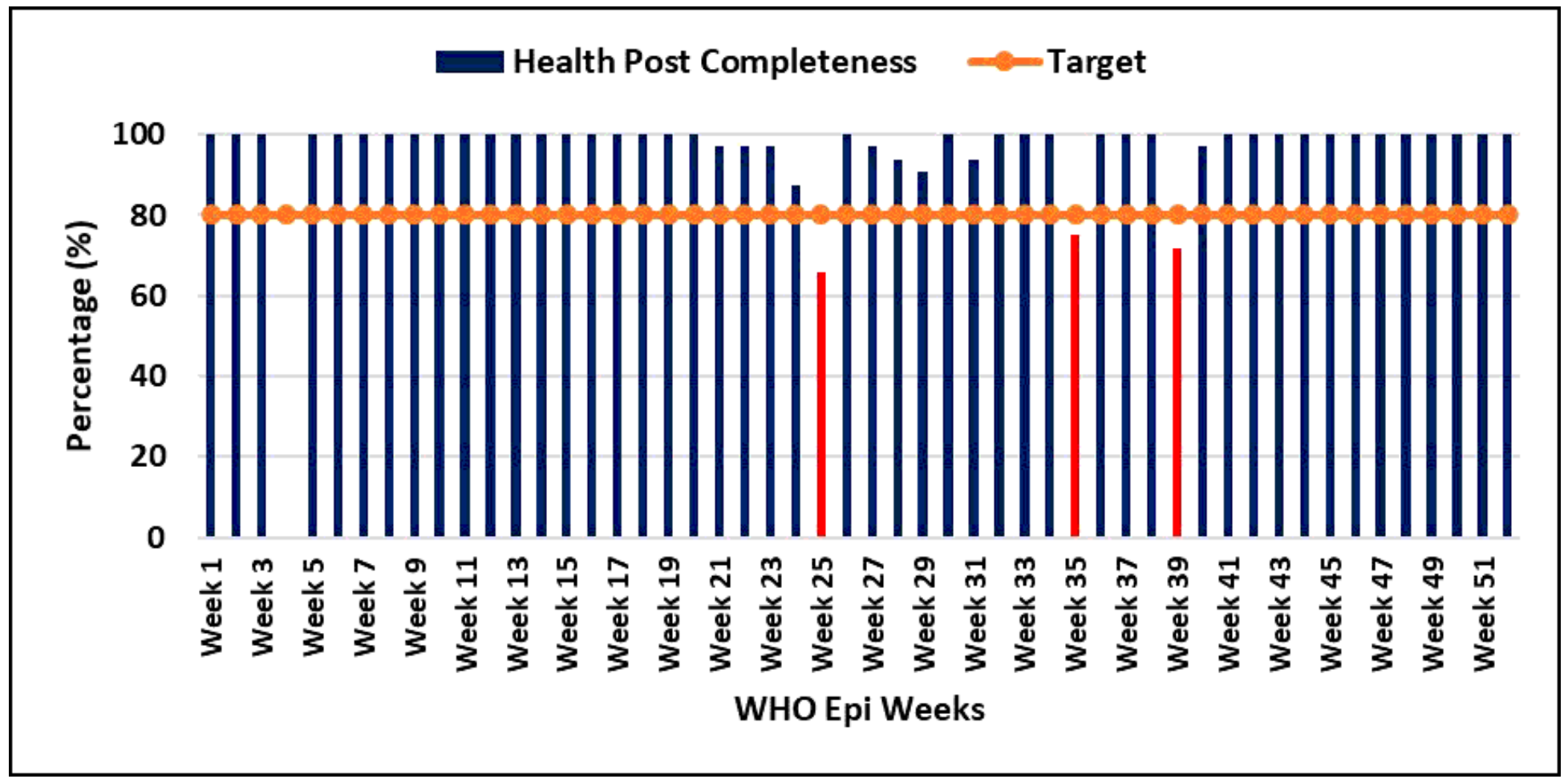


Figure 4

Completeness of health posts weekly surveillance report by WHO weeks, Ginnir district, Southeast Ethiopia.

Woreda Completeness

$\longrightarrow$ Target

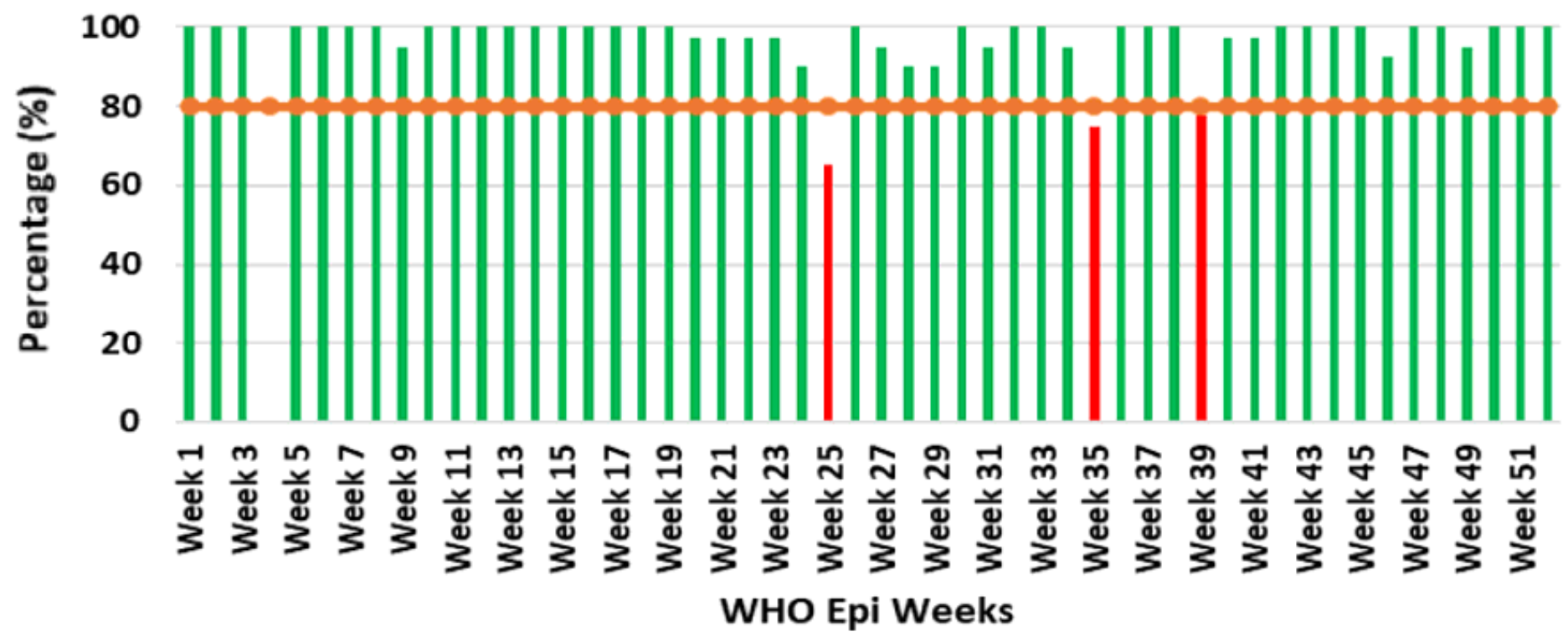

Figure 5

Overall completeness of weekly surveillance report by WHO weeks in Ginnir district, Southeast Ethiopia.

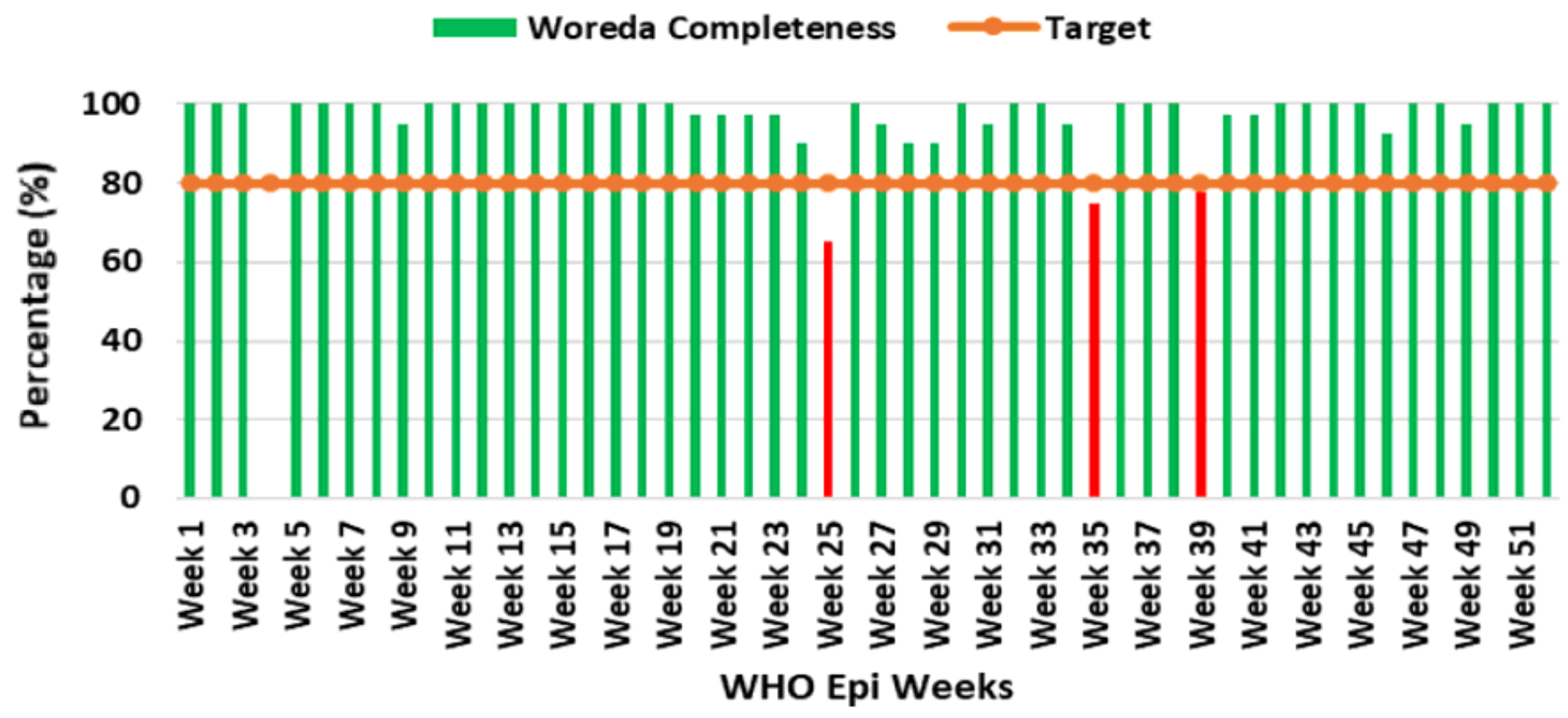

Figure 5 
Overall completeness of weekly surveillance report by WHO weeks in Ginnir district, Southeast Ethiopia.

\section{Supplementary Files}

This is a list of supplementary files associated with this preprint. Click to download.

- Revisedadditionalfile1.docx

- Revisedadditionalfile1.docx 